\title{
Dissociation of the N400 component between linguistic and non-linguistic processing: A source analysis study
}

\author{
Anne Gallagher ${ }^{1,2}$, Renée Béland ${ }^{2}$, Phetsamone Vannasing ${ }^{1}$, Maria Luisa Bringas ${ }^{3}$, Pedro Valdes Sosa ${ }^{4}$, \\ Nelson J. Trujillo-Barreto ${ }^{4}$, John Connolly ${ }^{5}$, Maryse Lassonde ${ }^{1,2}$ \\ ${ }^{1} \mathrm{CHU}$ Ste-Justine Research Center, Montreal, Canada \\ ${ }^{2}$ Centre de Recherche en Neuropsychologie et Cognition, Psychologie Department, University of Montreal, Montreal, Canada \\ ${ }^{3}$ International Center for Neurological Rehabilitation (CIREN), Havana, Cuba \\ ${ }^{4}$ Centro de Neurosciencias, Havana, Cuba \\ ${ }^{5}$ Language, Memory \& Brain Laboratories, Department of Linguistics \& Languages, McMaster University, Hamilton, Canada \\ Email: anne.gallagher@umontreal.ca
}

Received 10 October 2013; revised 10 November 2013; accepted 18 November 2013

Copyright (c) 2014 Anne Gallagher et al. This is an open access article distributed under the Creative Commons Attribution License, which permits unrestricted use, distribution, and reproduction in any medium, provided the original work is properly cited. In accordance of the Creative Commons Attribution License all Copyrights (C) 2014 are reserved for SCIRP and the owner of the intellectual property Anne Gallagher et al. All Copyright (C) 2014 are guarded by law and by SCIRP as a guardian.

\section{ABSTRACT}

The N400 component is commonly associated with the detection of linguistic incongruity. A few studies have shown that the $\mathbf{N 4 0 0}$ can also be elicited by non-linguistic stimuli. Different spatiotemporal patterns were observed between the typical Linguistic N400 and the Non-linguistic N400, suggesting distinct brain generators. The aim of this study was to investigate the presence of an $\mathrm{N400}$ in response to linguistic and non-linguistic stimuli, and to specify anatomical sources of both N400s using a novel analysis method: the Bayesian Model Averaging (BMA) distributed source model. Picture-word and environmental soundpicture associations, either congruent or incongruent, were presented to ten young healthy adults while highdensity ERP recordings were made. Standard electrophysiological analyses confirmed that the $\mathrm{N400}$ was not specific to linguistic incongruity but was also elicited by environmental sound-picture incongruities. Different topographic distributions were obtained for the Linguistic N400 and Non-linguistic N400. BMA analysis showed that the Linguistic $\mathbf{N 4 0 0}$ generators were mostly located in the left superior temporal gyrus, whereas the sources of the Non-linguistic N400 were identified mostly in the right middle and superior temporal gyri. Detection of linguistic incongruities recruited cerebral areas commonly associated with language processing, whereas non-linguistic incongruities recruited right cerebral regions usually associated with auditory processing of non-linguistic stimuli. The Linguistic and Non-linguistic N400s appear to be elicited by similar cognitive mechanisms assumed by different cerebral areas depending on the type of material to be processed. The present findings support the existence of parallel pathways for the processing of linguistic and non-linguistic incongruities.

\section{KEYWORDS}

Event-Related Potentials; Incongruity; Semantic; Environmental Sounds; Distributed Source Model; BMA

\section{INTRODUCTION}

Event-related potentials (ERPs) provide an online measure of cortical activity [1] and have been useful in studying cognitive processes. Some ERP components, such as the N400, can provide specific information associated with semantic processing. The N400 is a negative deflection, which peaks between 350 and $600 \mathrm{~ms}$ after stimulus onset [2-4]. This component was originally observed when subjects read sentences that ended with semantically incongruent words (for example: "I take my coffee with cream and engine") [5,6]. The N400 would then reflect the participant's reprocessing of the unusual sentence ending. Kutas and Hillyard [7] further showed that the degree of unpredictability, and not only that of semantic incongruity, could increase the amplitude of the N400 (for example: "The bill was due at the end of the hour").

The N400 was also elicited in tasks involving the presentation of word pairs presented either visually or aurally [8,9], word-picture pairs [10], and word-iconic 
gesture pairs [11]. For instance, Connolly et al. [10] using a computer-based adaptation of the Peabody Picture Vocabulary Test-Revised (PPVT-R) [12], presented a picture and an auditory word stimulus with a $700 \mathrm{~ms}$ onset asynchrony. Half of the picture-word pairs were correctly associated (e.g. the auditory word stimulus "ball" presented with the picture of a ball), whereas the other half of pairs were incorrectly associated (e.g. the auditory word stimulus "car" presented with the picture of a ball). Incorrectly associated pairs elicited significantly larger N400 amplitudes than correctly associated pairs. A French adaptation of this task was developed for the present study.

Although the vast majority of the studies on the N400 were carried out in the language domain, the N400 component can also be elicited in response to detection of incongruity involving non-linguistic meaningful stimuli, such as line drawings [13,14], photographs [15], faces [16-19], arithmetic equations [20,21], environmental sounds [22-30], and even odors [31,32]. Interestingly, different spatiotemporal patterns were observed for the typical Linguistic N400 and the Non-linguistic N400. The Linguistic N400 component is defined as a centroparietal negative wave $[5,33]$, whereas the Non-linguistic N400 is defined as a fronto-central negative component $[13,14,22,30]$. A right-greater-than-left asymmetry was associated with the Linguistic N400 [5,33], whereas the opposite left-greater-than-right asymmetry was reported for the Non-linguistic N400 [22,26,27,30,34]. As for the differences in the temporal pattern, a shorter latency was observed for the Non-linguistic N400 compared to the

\section{Linguistic N400 [22,23,27].}

Source localization (or source reconstruction) enables the investigation of the neural generators of ERP components. Localization of these generators involves addressing the EEG Inverse Problem. This consists in the estimation of the distribution of the Primary Current Density (PCD) inside the brain that produces a given measured EEG. There are infinite solutions to this problem and thus specific additional prior information (or constraints) about the EEG generators must be included in order to obtain a unique and physiologically valid solution. Depending on the type of prior information used, there is a whole spectrum of different methods available.

Some methods handle the many-to-one nature of this problem (many parameters to estimate and very few data available) by characterizing the sources in terms of a limited number of current dipoles (dipolar model) that are fitted to the data through the minimization of some measure of the reconstruction error [35-38]. In such models, each dipole represents the summed activity of a small cortical region and assumes that these dipoles vary only in strength over time [39]. Over the last fifteen years, a number of studies attempted to localize the generators of the Linguistic N400 using different models for source localization. A summary of the results of these studies is reported in Table $\mathbf{1}$. For example, using a dipolar model approach, three MEG studies identified a left postero-superior temporal lobe source for the Linguistic N400 [40-42] while a fourth MEG study [43] reported bilateral auditory cortex generators for the same component. EEG studies using a dipolar model localized the

Table 1. Linguistic N400 source analysis literature review (in alphabetic order) from dipole and distributed source models. Lt = left; Rt $=$ right.

\section{Previous studies}

D’Arcy et al. 2004 [44]

D’Arcy et al. 2005 [45]

Dien et al., 2010 [46]

Hamm et al. 2002 [47]

Helenius et al. 1998, 1999 [40,41]

Mäkela et al. 2001 [43]

Silva-Pereyra et al. 2003 [48]

Simos et al. 1997 [42]
Linguistic N400 cerebral generators

Dipole model

Lt perisylvian cortex
Lt perisylvian cortex
Bilateral anterior medial temporal lobe
Rt frontal and bilateral temporal generators
Lt postero-superior temporal lobe
Bilateral auditory cortex generators
Bilateral inferior, middle, and superior temporal regions, inferior frontal
and insular areas, anterior cingulated, occipito-temporal and occipital areas
Lt postero-superior temporal lobe
Distributed source model
Lt temporal lobe
Lt temporal pole, Lt supramarginal gyrus and Lt inferior frontal gyrus
No specific localization
Wernicke’s area (Lt hemisphere)
Bilateral middle/superior temporal gyrus
Lt and Rt fusiform gyrus, Lt cingulate gyrus, Rt limbic area, Rt middle temporal gyrus and Lt middle frontal gyrus
Lt medial frontal area
Left anterior source localization
Lt fronto-temporal areas

Bölte et al., 2010 [49]

Giglio et al., 2013 [50]

Haan et al. 2000 [51]

Halgren et al. 2002 [52]

Khateb et al. 2007, 2010 [53,54]

Proverbio et al. 2009 [55]

Qiu et al. 2008 [56]

Schulz et al. 2008 [58]

Travis et al., 2013 [59] 
sources of the Linguistic N400 along the left perisylvian cortex [44,45], bilateral anterior medial temporal lobe [46] or in bilateral superior temporal gyrus [47], while Silva-Pereyra et al. [48] reported a very wide distribution of Linguistic N400 sources in bilateral inferior, middle, and superior temporal regions, inferior frontal and insular areas, anterior cingulate as well as occipito-temporal and occipital areas.

The growing experimental evidence about the existence of more diffuse brain networks has led to the emergence of a second method for source localization: the so-called distributed inverse solutions (DIS). These solutions address the non-uniqueness of the EEG inverse problem by using both anatomical and mathematical constraints. The anatomical constraints assume that the EEG sources are located in those brain regions capable of generating voltage fields over the scalp surface (gray matter for example), while the mathematical constraints make physiologically based assumptions about the mathematical properties of the solution obtained (like maximum spatial smoothness as in LORETA, for more details see [60] Trujillo-Barreto et al., 2004).

Several studies reported in Table 1 used a DIS approach to localize the sources of the Linguistic N400. For instance, Halgren et al. [52] visually presented sentences ending with semantically congruous or incongruous words to healthy adults during MEG recording. They found a Linguistic N400 occurring at around 250 ms that originated from the left Wernicke's area, lying in or near the superior temporal sulcus. The N400 activation then spread at about $270 \mathrm{~ms}$ to the anterior temporal sites and later on, at about $300 \mathrm{~ms}$, to Broca's area and, finally, at about $370 \mathrm{msec}$, to the anterior orbital and fronto-polar cortices. In another study, Schulz et al. [58] who used LORETA and LAURA source localization algorithms with EEG data, reported that the generators of the Linguistic N400 elicited by their sentence-reading task were predominantly located in the left anterior middle temporal area. Similarly, Giglio et al. [50] and Travis et al. [59] found a left fronto-temporal N400 source distribution using distributed source models. However, Khateb et al. [53] investigated, using LAURA, source localization of N400s elicited from different linguistic tasks including different stimulus types (words vs. images) and showed the involvement of the bilateral middle/superior temporal gyrus in all tasks. Hamm et al. [47], who used the LORETA source localization algorithm, reported right frontal and bilateral temporal generators, whereas Haan et al. [51] found a very broad and scattered distribution for the generators of the Linguistic N400, which they attributed to important inter-individual differences impeding-accurate localization of the sources.

To sum up, investigation of the Linguistic and Nonlinguistic N400 components revealed differences in their spatial distribution. For the Linguistic N400, findings of the studies reported in Table 1 show a tendency to localize more commonly sources of the Linguistic N400 in the left temporal lobe. Nonetheless, the results regarding the localization of the generators of the Linguistic N400 from these studies show some inconsistencies. The discrepancy may stem, either from differences in the tasks that were used to elicit the Linguistic N400, some of these tasks included linguistic and non-linguistic stimuli within the same experimental paradigm, or from differences in the models and algorithms that were used for source localization. Development of more reliable and adapted DIS for late component such N400 is thus needed.

To our knowledge, no electrophysiological studies have so far been conducted in order to localize the cerebral generators of the Non-linguistic N400 elicited by environmental sounds. The only source of information comes from a study by Kotz et al. [61] who reported that a N400 was elicited by meaningful environmental sounds in healthy adults but not in patients with either left or right anterior temporal lobe lesion. The present set of experiments aims to clarify whether or not the Linguistic and the Non-linguistic N400 are distinct components generated by different groups of neurons. Among the non-linguistic sounds, environmental sounds are those that received the most attention. Environmental sounds are construed as the best non-linguistic stimuli homologous to human speech because they have a complex spectral structure, and they are easily identifiable [62]. Results of the studies conducted with brain-damaged patients [63] as well as with normal subjects using fMRI [64] or PET [62,65,66] suggested that environmental sounds are mostly processed in the right hemisphere. Environmental sounds are thus well-suited stimuli to use in a task eliciting a Non-linguistic N400, because the generators of this component are likely to be located in the right hemisphere, in contrast with the Linguistic N400 whose generators are generally located in the left hemisphere [40,42,44,52].

The present study constitutes the first attempt to investigate anatomical sources of both the Linguistic N400 and Non-linguistic N400 (elicited by an environmental sound-picture task). To this end, picture-auditory word and environmental sound-picture pairs that were erroneously or correctly matched were presented to participants during ERP recording. Source analyses based on a distributed Bayesian model averaging (BMA) approach [60] were performed on both N400 components.

The BMA approach is based on the application of the Bayesian model inference scheme [67] using evidence approximation [68], to the EEG/MEG Inverse Problem. It has been used recently by Olivares and colleagues [19] to localize a N400-like effect elicited by a familiar faces 
matching task. The Bayesian paradigm allows estimating the posterior probability of each model given the data, which represents a measure of "how good" that model is for explaining the data. This measure of goodness expresses a trade off between goodness-of-fit (data reconstruction error based on that model) and complexity (number of parameters used by the model to explain the data) of the model. The number of parameters of the given model is proportional to the total number of voxels contained in the anatomical regions used to constrain the inverse solution in that model. That is, higher probabilities are assigned to the simpler models that explain the data better. Then, the model uncertainty is taken into account by averaging the primary current densities (PCDs) obtained for each particular anatomical constraint (or model) weighted by their model posterior probabilities. This weighted average provides a PCD, which is unconditional on any of the anatomical constraints assumed. In this approach, the solution under each anatomical constraint is obtained using the traditional LORETA method [69].

\section{MATERIALS AND METHODS}

\subsection{Participants}

The sample was composed of 10 young healthy adults (five males, age range $=20-28$ years with a mean age of 24.78 years). All participants were native speakers of Canadian French. The data from one male participant had to be rejected because of electrophysiological artefacts. Informed consent was obtained from all participants prior to their inclusion in the study. The project was approved by the Ethics Committees of the SainteJustine and Notre-Dame University of Montreal Hospital Centers. Moreover, all participants gave their informed consent prior to their inclusion in the study.

\subsection{Tasks and Stimuli}

All participants performed two tasks 1) the environmental sound-picture matching task, which consisted of determining whether a presented picture was congruent or not to an environmental sound (non-linguistic task) and 2) the picture-auditory word matching task, consisting in determining whether an auditory word was congruent or not to a picture (linguistic task).

\subsubsection{Environmental Sounds}

The 180 environmental sounds (for example, bird songs, car noises, waterfall sounds, etc.) used in this study were taken from the Sounds Effects Library created by LucasFilm Ltd \& Sound Ideas. Onset and offset of each sound was determined using Cool Edit Pro ${ }^{\mathrm{TM}}$ software allowing the creation of sounds that lasted exactly $2383 \mathrm{~ms}$. Environmental sounds were presented with Optimus 40232 model XTS24 speakers at $75 \mathrm{~dB}$. Ninety environmental sounds were correctly associated to a picture (e.g. the noise of a truck paired with the picture of a truck) and 90 others were incorrectly paired to the same set of pictures (e.g. a laugh, paired with the picture of a truck).

\subsubsection{Words}

All 180 words were digitally recorded in stereo in a male voice using Cool Edit Pro ${ }^{\mathrm{TM}}$ software. Word duration varied from 327 to $1104 \mathrm{~ms}$, with a mean duration of 684 ms. Onset and offset of each word were determined visually with PRAAT ${ }^{\mathrm{TM}}$ software based on the word's digitized waveform, allowing precise cutting of each word. Words were presented with Optimus 40232 model XTS24 speakers at $75 \mathrm{~dB}$. The 90 auditory words correctly associated with a picture were taken from the Échelle de Vocabulaire en Images Peabody (EVIP) [70]. The 90 words that were erroneously matched to the same set of pictures were selected from different word lists [71-74]. They were paired with the set of congruent words based on acquisition age, number of syllables, lexical category, and the phonetic features of the first phoneme, which was either a voiced or unvoiced stop (e.g. b, t) or voiced or unvoiced fricative (e.g. v, f). In the incongruent pairs, the auditory word and the picture did not belong to the same semantic category nor did they share phonological similarity. For instance, the picture of a boat (the French word for boat is "bateau") was paired with the heard word "gorille" (meaning "gorilla" in English).

\subsubsection{Pictures}

All 180 pictures paired with auditory stimuli (environmental sounds and words) were simple black and white drawings of $420 \times 315$ pixels (a visual angle of approximately 7 degrees) taken from the EVIP, the French version of the PPVT-R, and from various web sites. Pictures derived from EVIP A and B booklets were selected among items from lowest performance levels and were electronically digitized with ScanJet 5300C $\mathrm{HP}^{\mathrm{TM}}$ and PrecisionScan ${ }^{\mathrm{TM}} 3.03$ software. All pictures were resized to a $1024 \times 768$ pixels resolution using Adobe Photoshop software and were presented in the middle of a ViewSonic Professional Series P225F screen (refreshing rate of $75 \mathrm{~Hz}$ ), which was placed $85 \mathrm{~cm}$ from the subject. Timing and sequence of pictures were controlled by a computer using MatLab 6.5 software and auditory stimuli were presented by a Tucker-Davis System (RP2.1; Real-time Processor). In each task, all pictures were presented twice because the same set of pictures was used for both congruous and incongruous pairs.

Pilot studies were performed to ensure that both tasks were successfully performed by participants (success rate over 90\%) and induced a N400 effect. These studies in- 
dicated that the N400 was best elicited with the sound preceding the picture in the environmental sound-picture matching task and the opposite was found for the picture-auditory word matching task. Figure 1(a) illustrates the procedure used in the environmental sound-picture matching task. Ninety pictures were paired with a congruent and an incongruent environmental sound. Each trial consisted of an environmental sound, which appeared $1400 \mathrm{~ms}$ before the picture onset and remained audible during picture presentation and 483 ms after the offset of the picture. The duration of the picture presentation was $500 \mathrm{~ms}$. A trigger was placed at the beginning of the presentation of each picture and was used to time-lock EEG sampling to picture onset.

In the picture-auditory word matching task (see Figure 1(b)), another set of 90 pictures was paired with a congruent and an incongruent word. Each trial consisted of a picture, which appeared $700 \mathrm{~ms}$ before the onset of the auditory word and remained visible during the auditory word presentation (mean duration was $684 \mathrm{~ms}$ ) and
$1000 \mathrm{~ms}$ after the offset of the word. A trigger was placed at the beginning of each word and was used to time-lock EEG sampling to word onset.

The Linguistic N400 is elicited by auditory stimuli (picture-auditory word matching task) whereas the Non-linguistic N400 is evoked by pictorial material (environmental sound-picture matching task). In previous studies, both pictorial stimuli and auditory stimuli have been found to elicit similar N400-like effects in response to semantic mismatch [15]. Nevertheless, a paired Hotelling T2 test (incongruent versus congruent) was applied on the data of each task in order to eliminate common and other early components when performing source analyses (see extended description in the Source Analyses Section).

\subsection{Procedure}

Participants performed both the picture-auditory word matching and environmental sound-picture matching tasks in dim light while sitting in a comfortable chair in a
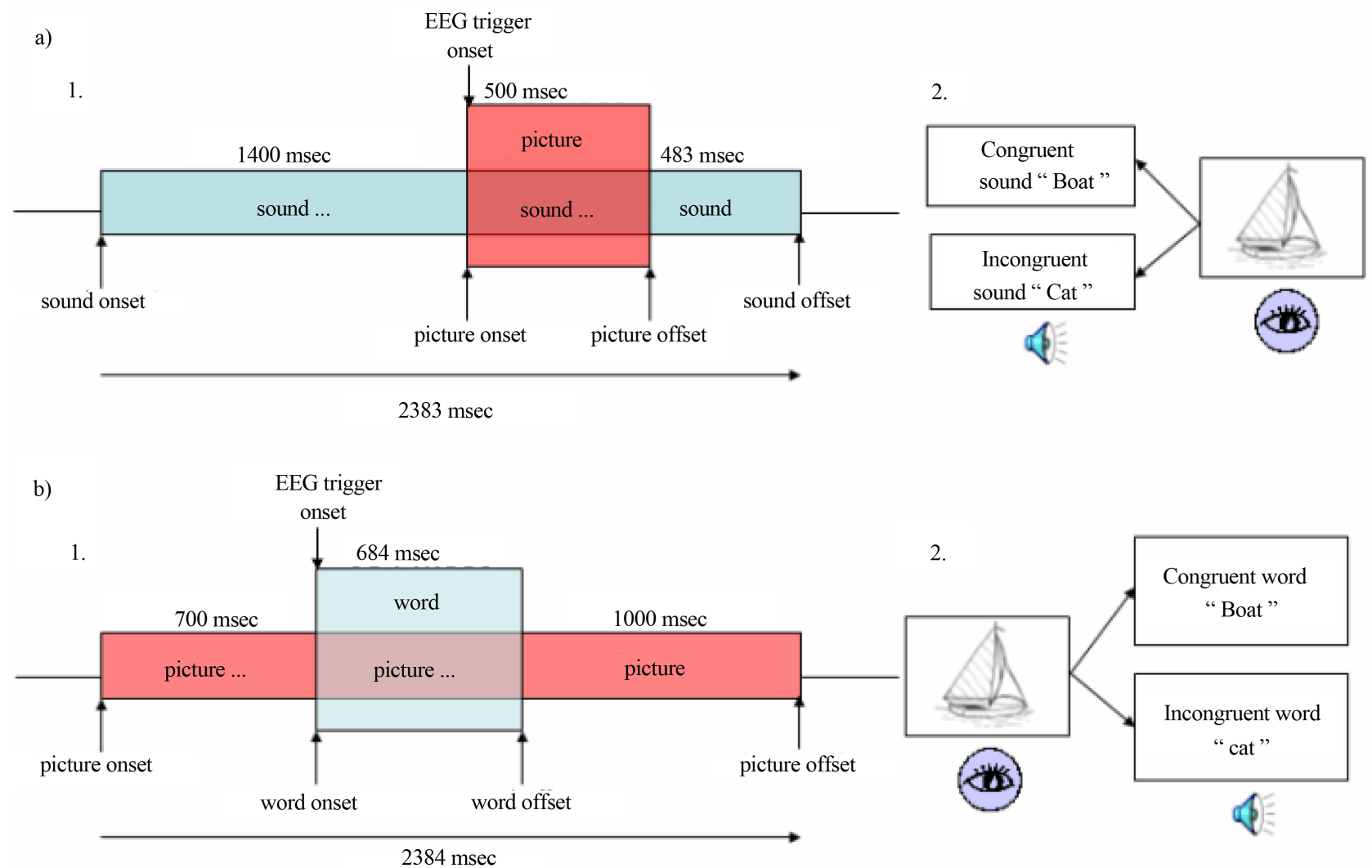

Figure 1. Procedure used in both tasks. (a) Environmental sound-picture matching task. (a1) Each trial consisted of a sound (blue rectangle), which appeared 1400 ms before picture onset. Every picture appeared on the screen for a duration of 500 ms (red rectangle). The sound remained on for the entire trial for a total trial duration of $2383 \mathrm{~ms}$. (a2) Each of the 90 pictures was correctly or erroneously paired with a sound. For example, for a congruent trial, the sound of a "boat" would be paired with a picture of a "boat" and for an incongruent trial the sound of a "cat" would be paired with the same picture of a "boat"; (b) Picture-auditory word matching task. (b1) The picture (red rectangle) appeared $700 \mathrm{~ms}$ before the word onset and remained visible during the entire trial (mean total trial duration of $2384 \mathrm{~ms}$ ). Word mean duration was $684 \mathrm{~ms}$ (blue rectangle). (b2) Each of the 90 pictures was correctly or erroneously associated to a word. 
sound-proof, electrically shielded room. Both tasks were composed of 4 practice trials followed by 180 experimental trials, and were administered during electrophysiological recording (see "Electrophysiological recording" section). The order of trial presentation was pseudo-randomized for both tasks; all trials were presented randomly with the exception of trials with the same picture, which were separated by at least one other trial. A 2-second inter-trial interval consisted of a blank screen with a central fixation cross ("“+”). Tasks were divided in four blocks of 45 trials, for a total of 180 experimental trials for each task. Since each trial had a duration of $4383 \mathrm{~ms}$, each block had a total duration of approximately 197 seconds (approximately 3 minutes 29 seconds). Short periods of rest (1 - 2 minutes) were given to participants between blocks to reduce fatigue and eye strain. All participants performed both tasks twice in order to increase the total number of trials from 180 to 360 in each task, for a total duration of approximately 60 minutes including inter-block and inter-task periods of rest. The total testing duration did not exceed two and a half hours including electrode placing, experiment, and electrode location digitalization. During the placing of the electrodes and the subsequent digitalization, which both took approximately 60 minutes; the participant was quietly watching a movie.

During both tasks, the participant responded bimanually on a keyboard to each trial by pressing two green buttons when the auditory and visual stimulus association was congruent, and by pressing two red buttons when the association was incongruent. A bimanual response was selected in order to eliminate unilateral cortical activation that would have been associated with mono-manual responses. All participants were asked to avoid blinking while the sound was audible (environmental sound-picture matching task) or the picture was visible (picture-auditory word matching task), and to blink between trials, if needed.

The environmental sound-picture matching task was administered prior to the picture-auditory word matching task in order to prevent as much as possible, the subjects from verbalizing the visual and auditory stimuli of the environmental sound-picture matching task. Indeed, the picture-auditory word matching task involved heard words that automatically recruit language processing. Hence, the administration of the picture-auditory word matching task prior to the environmental sound-picture matching task would likely have accustomed and encouraged the participant to adopt a linguistic strategy in performing the subsequent environmental sound-picture matching task.

\subsection{ERP Recordings and Analyses}

EEG activity was recorded from $128 \mathrm{Ag} / \mathrm{AgCl}$ electrodes with a NeuroScan Synamps $2^{\mathrm{TM}}$ system at a sampling rate of $500 \mathrm{~Hz}$ with a bandpass filter of $0.1-100 \mathrm{~Hz}$ and a central reference. The electrodes were embedded in a 128 NeuroScan Quick-Cap ${ }^{\mathrm{TM}}$. Impedances were maintained at or below $5 \mathrm{k} \Omega$ during the whole recording session and an electrode placed in the frontal area (between $\mathrm{AFz}$ and $\mathrm{Fz}$ ) served as ground. Vertical and horizontal eye movements were recorded using external electrodes placed above, under, and on the outer canthus of both eyes. Heart rate and muscular activity were also recorded from, respectively, left and right electrodes placed on Erb point and on both sides of the neck. Evoked potentials were recorded with Neuroscan 4.3 software (Compumedics, USA).

Location of each electrode and three fiducial points (nasion, and left and right preauricular) was digitized and recorded with Brainsight ${ }^{T M}$ Frameless system (Rogue Research, Montreal, Canada) on a Macintosh G4 computer to allow for source analysis.

EEG recordings were analysed using the Brain Vision Analyser program (Brain Products, Munich, Germany). A digital band pass filter of $0.1-30 \mathrm{~Hz}$ and $24 \mathrm{~dB}$ /octave, as well as ocular correction [75] were applied off-line. Data were referenced to the averaged signal of all electrodes. The continuous EEG recordings were then epoched beginning $200 \mathrm{~ms}$ before, and ending $1000 \mathrm{~ms}$ after stimulus onset (601 data points). Segmented data were corrected using an artefact rejection (threshold of $\pm 100 \mu v$ ). Data were averaged across trials for each experimental condition after baseline correction using the 200-ms time segment before stimulus onset.

The N400 component was scored as the most negative peak occurring between 325 and $475 \mathrm{~ms}$ for the environmental sound-picture matching task and between 375 and $500 \mathrm{~ms}$ for the picture-auditory word matching task. N400 peak amplitudes were measured as the voltage difference between the mean activity for the 200-ms baseline period before stimulus onset and the most negative point within the specified latency range. N400 peak latencies corresponded to the time from stimulus onset and to the most negative point within the specified latency range. Topographical maps (Brain Vision) were computed from the normalized amplitude data [76,77].

\subsection{Source Analyses}

EEG data collected from each task (linguistic and nonlinguistic) and condition (incongruent and congruent) were processed separately. After averaging over all accepted trials, the specific N400 peak latency for each subject was identified as described in the previous section. Source analyses were performed individually at the N400 peak latency, on each task and condition separately using BMA approach.

An image of the underlying brain electrical activity 
(primary current density) for each individual scalp topography, for each task and condition, was estimated by BMA of different EEG inverse solutions (see [60] for details), each one based on anatomical constraints derived from the Montreal Neurological Institute MNI average-brain atlas. Individual lead fields were calculated using the Reciprocity Theorem [78], with a three spheres and isotropic conductivities head model [79,80]. A constraint-independent final image of the underlying brain electrical activity related to each task and each condition was then obtained by averaging all computed primary current densities weighted by the corresponding support that they received from the data. This weighting coefficient was measured in terms of the posterior probability of the corresponding model (anatomical constraint), given the data. In order to measure statistical differences between both incongruent and congruent conditions of the currents estimated $[(\mathrm{x}, \mathrm{y}, \mathrm{z})$ components] at each voxel, we used paired Hotelling T2 statistic tests for each task separately. Based on previous peak detection analyses (see section "ERP analyses"), the paired Hotelling T2 statistic test was applied in a time window between 325 and $475 \mathrm{~ms}$ for the non-linguistic task and between 375 and $500 \mathrm{~ms}$ for the linguistic task. The threshold for the resulting statistic parametric map was selected using false discovery rate [81] with $\mathrm{q}=0.1$. This test provided inverse solution statistical maps comparing both conditions for each task separately. Individual and Grand Average inverse solutions were visualized using the Brain Electrical Tomography (BET) viewer software (Neuronic S.A.), which integrates the functional information provided by the EEG with the anatomical information provided by the MRI.

\section{RESULTS}

\subsection{Behavioral Analyses}

Success rates for both tasks were measured to ascertain that the participants' attention level was acceptable and that they were performing the task adequately. Individual success rates varied between $91.11 \%$ and $99.72 \%$ for the environmental sound-picture matching task, and between $96.39 \%$ and $100 \%$ for the picture-auditory word matching task. On average, participants could accurately recognize $96.98 \% \pm 3.22 \%$ of the congruent/incongruent stimulus pairs of the environmental sound-picture matching task and $98.73 \% \pm 1.63 \%$ of congruent/incongruent stimulus pairs during the picture-auditory word matching task. No difference was found between congruent and incongruent trials. Although a Student $t$ test for task difficulty difference did not reach significance $\left(\mathrm{t}_{9}=2.271, \mathrm{p}\right.$ $=0.053$ ), the performance during the picture-auditory word matching task tended to be slightly higher than that observed for the environmental sound-picture matching task.

Following testing, the participants were questioned to determine if they had used different strategies when performing the two tasks. They indicated that during the environmental sound-picture matching task, they tried to visualize the object corresponding to the sound they were hearing whereas during the picture-auditory word matching task, they silently anticipated the word corresponding to the picture being presented.

\subsection{Standard Electrophysiological Analyses}

Considering the high success rates obtained for both tasks, ERP analyses were conducted on all trials. Moreover, less than $10 \%$ of all trials were rejected due to artifact or ocular correction. Figure 2 shows the grand-average ERPs obtained when averaging the responses elicited by each stimulus for all participants during Figure 2(a) picture-auditory word matching tasks and Figure 2(b) environmental sound-picture.

On average, the N400 elicited by the environmental sound-picture matching task (Figure 2(b)) began at around 298 ms post-stimulus onset. The largest N400 amplitude was found at FCz and had an amplitude of $-3.603 \mu \mathrm{V}$ with a latency of $360 \mathrm{~ms}$ for the incongruent condition and an amplitude of $-2.638 \mu \mathrm{V}$ with a latency of $352 \mathrm{~ms}$ for the congruent condition. A fronto-central topographical distribution was observed for the Non-linguistic N400 (Figure 2(b)).

The N400 component waveform found in the picture-auditory word matching task (Figure 2(a)) began on average at approximately $342 \mathrm{~ms}$ post-stimulus onset. The largest N400 was found in $\mathrm{Cz}$ where the amplitude was on average $-1.485 \mu \mathrm{V}$ with a latency of $414 \mathrm{~ms}$ for the incongruent condition and $0.097 \mu \mathrm{V}$ with a latency of $416 \mathrm{~ms}$ for the congruent condition. The Linguistic N400 presented a centro-parietal distribution (Figure 2(a)).

Because previous work suggested that presentation of the same stimuli can reduce the amplitude and shorten the duration of the N400 (e.g. [82,83], split-half analyses on amplitude and latency data as well as on success rates were computed to address the issue of habituation. An ANOVA with time (1 versus 2 ) and condition (congruent versus incongruent) as factors revealed that N400 amplitudes and latencies did not differ between both administrations of the protocol $(p>0.1)$. Furthermore, performance did not improve with increased exposure to the task, as indicated by near zero statistical values for Time. Therefore, all 360 trials of both tasks were used in further analyses.

\subsubsection{N400 Latency}

The analysis of the N400 latencies was conducted on the ten midline electrodes because of its well-known centroparietal distribution of the N400 component (e.g. $[5,33])$. 

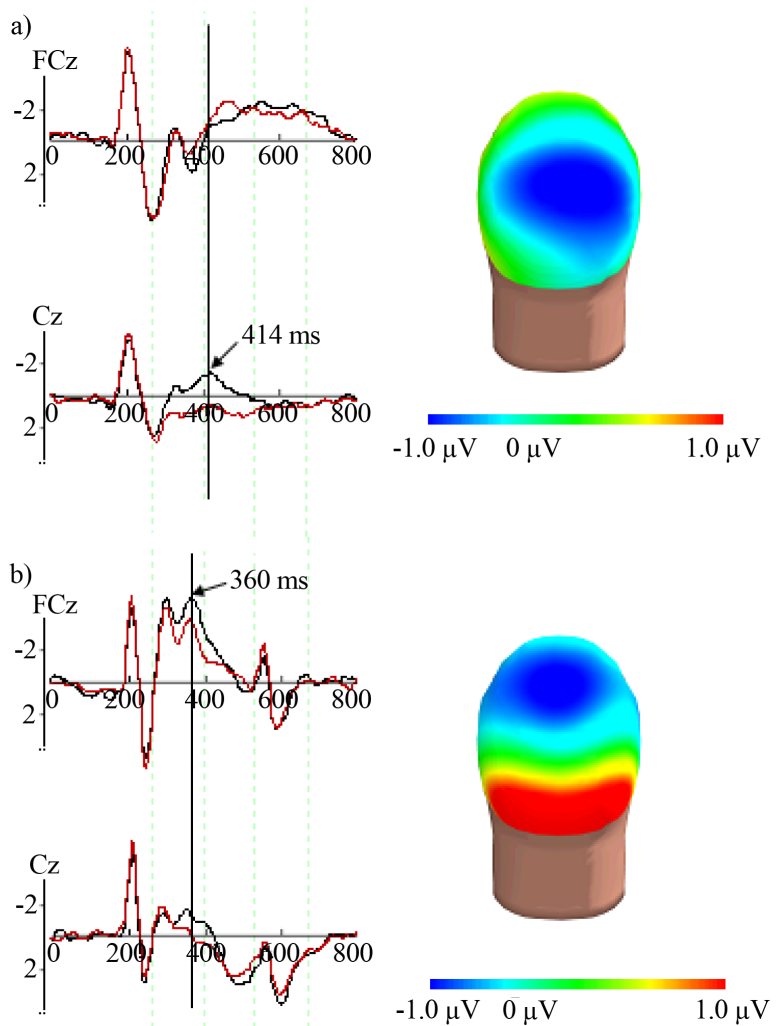

Figure 2. Grand-average ERPs obtained when averaging the responses elicited by each stimulus for all participants during both tasks. (a) Linguistic task. The largest Linguistic N400 was found in $\mathrm{Cz}$ where the amplitude was on average $-1.485 \mu \mathrm{V}$ with a latency of 414 ms for the incongrent condition (black line on the graph) and $0.097 \mu \mathrm{V}$ with a latency of $416 \mathrm{~ms}$ for the congruent condition (red line on the graph). As shown on the right (a), the Linguistic N400 presented a centro-parietal distribution; (b) Non-linguistic N400 and its topography. The largest Non-linguistic N400 amplitude was found at FCz and had an amplitude of $-3.603 \mu \mathrm{V}$ with a latency of $360 \mathrm{~ms}$ for the incongruent condition (black line on the graph) and an amplitude of $-2.638 \mu \mathrm{V}$ with a latency of $352 \mathrm{~ms}$ for the congruent condition (red line on the graph). A fronto-central topographical distribution was observed for the Non-linguistic N400 (right b). In order to get a better display, only most relevant electrodes for both N400 are shown (FCz and Cz).

A three-way within-subject ANOVA with task (non-linguistic versus linguistic), condition (congruent versus incongruent), and region (average of the five most anterior midline electrodes covering frontal and fronto-central areas versus average of the five most posterior electrodes placed on centro-parietal and occipital regions) as factors was performed on the N400 latency data. This analysis revealed a main effect of task $\left(\mathrm{F}_{(1,8)}=41.70\right.$, $\mathrm{p}<$ $0.001, \varepsilon=1.00)$ due to shorter Non-linguistic N400 latencies in response to pictures in the environmental sound-picture matching task (mean $=351.56 \pm 27.70 \mathrm{~ms}$ ) in comparison to Linguistic N400 latencies in response to auditory words presented in the picture-auditory word matching task (mean $=422.17 \pm 33.15 \mathrm{~ms}$ ). No interactions or other simple effects were found.

\subsubsection{N400 Amplitude}

A three-way within-subject ANOVA with task (non-linguistic versus linguistic), condition (congruent versus incongruent), and region (anterior versus posterior) as factors was also performed on the N400 amplitude data derived again from the 10 midline electrodes. This ANOVA revealed an effect of the condition factor $\left(\mathrm{F}_{(1,8)}\right.$ $=18.95, \mathrm{p}<0.01, \varepsilon=0.966)$, an effect of region $\left(\mathrm{F}_{(1,8)}=\right.$ 10.14, $\mathrm{p}<0.05, \varepsilon=0.796)$, an interaction task $\mathrm{x}$ region $\left(\mathrm{F}_{(1,8)}=13.90, \mathrm{p}<0.01, \varepsilon=0.902\right)$, a condition $\mathrm{x}$ region interaction $\left(\mathrm{F}_{(1,11)}=8.94, \mathrm{p}<0.05, \varepsilon=0.745\right)$ as well as a 3-way task $\mathrm{x}$ condition $\mathrm{x}$ region $\left(\mathrm{F}_{(1,8)}=12.97, \mathrm{p}<0.01\right.$, $\varepsilon=0.882)$ interaction. Decomposition of this 3-way interaction revealed that Non-linguistic N400 amplitudes related to the environmental sound-picture matching task were significantly larger in the anterior (congruent = $-2.45 \mu \mathrm{V}$; incongruent $=-3.30 \mu \mathrm{V}$ ) than the posterior (congruent $=1.01 \mu \mathrm{V}$; incongruent $=0.47 \mu \mathrm{V}$ ) regions for both the incongruent $\left(t_{9}=-3.601, p=0.007\right)$ and congruent $\left(\mathrm{t}_{9}=-3.498, \mathrm{p}=0.008\right)$ conditions, again reflecting the anterior treatment of non-linguistic incongruity. In contrast, Linguistic N400 amplitudes related to the picture-auditory word matching task were significantly larger $\left(\mathrm{t}_{9}=4.22, \mathrm{p}=0.003\right)$ in anterior than posterior regions for congruent stimuli (anterior $=-1.27 \mu \mathrm{V}$; posterior $=0.53 \mu \mathrm{V}$ ) whereas no significant differences were found between anterior and posterior regions for incongruent stimuli $\left(\mathrm{t}_{9}=-0.955, \mathrm{p}=0.368\right.$; anterior $=-0.61$ $\mu \mathrm{V}$; posterior $=-1.23 \mu \mathrm{V}$ ).

In order to further compare topographical data between both tasks, a $t$-test was conducted using BrainVision on normalized amplitude data [76,77]. Given the Student's $t$-distribution table, it was assumed that in order for activity distribution to be significantly different across both tasks in the incongruent condition, the $t$ value would have to be $>3.36$. The two N400 component distributions were spatially different $\left(\mathrm{t}_{8}>3.36, \mathrm{p}<0.05\right)$ between 340 and 430 ms: the Non-linguistic N400 (environmental sound-picture matching task) was associated with a fronto-central scalp distribution whereas the Linguistic N400 (picture-auditory word matching task) produced a centro-parietal distribution. This significant time window (340 - $430 \mathrm{~ms}$ ) has been further used for source analyses.

With the aim of investigating the presence of hemispheric asymmetries, a $t$-test was performed. Consistent with the literature, hemispheric asymmetries were found for amplitudes in the picture-auditory word matching task $\left(\mathrm{t}_{8}=2.788, \mathrm{p}=0.024\right)$, where Linguistic N400 amplitudes were larger on the right hemisphere (an average of the data from 20 electrodes overlying the right fron- 
to-centro-parietal area) than on the left hemisphere (an average of the data from 20 electrodes overlying the left fronto-centro-parietal area). No hemispheric asymmetries were found in the Non-linguistic N400 elicited by the environmental sound-picture matching task.

\subsection{Electrophysiological Source Analyses}

In order to determine if the N400 components elicited by the picture-auditory word and environmental sound-picture matching tasks had different N400 generator localizations, source analyses using a distributed BMA approach were performed on both N400s. Results suggested different cerebral sources for each component elicited by incongruous trials. In fact, the generators of the Non-linguistic N400 were situated in the right middle and superior temporal gyri, in bilateral parahippocampal gyri and in the left lingual gyrus (Figure 3(a)), whereas those for the Linguistic N400 were located in the left superior temporal gyrus and in the right parahippocampal

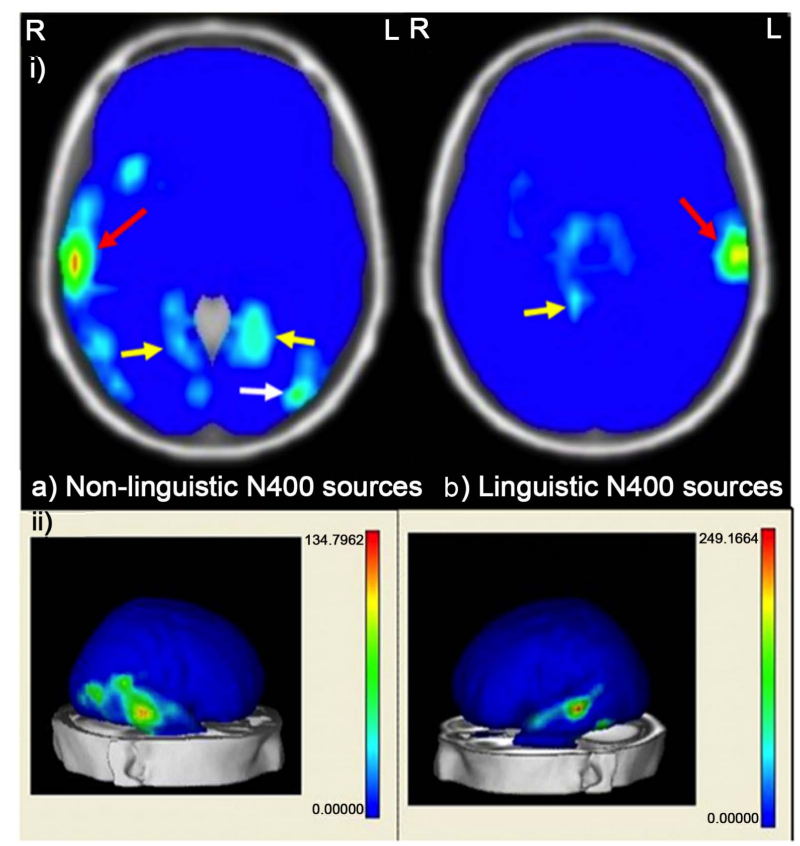

Figure 3. BMA source analyses results for (a) environmental sound-picture matching task and (b) picture-auditory word matching task. All individual inverse solutions have been average in order to visualize grand-average BMA source analyses. Results show (a, i) right (R) middle and superior temporal gyri (red arrow), bilateral parahippocampal gyri (yellow arrows) and left (L) lingual gyrus (white arrow) sources for the Nonlinguistic N400. Left ii) shows right (R) middle and superior temporal (auditory area) Non-linguistic N400 generators on a 3D brain reconstruction; (b) L superior temporal gyrus (red arrow) and R parahippocampal gyrus (yellow arrow) generators for the Linguistic N400. Right ii) shows clear L superior temporal (Wernicke's area) Linguistic N400 generators. N.B. As specified on the figure, the radiological convention is used: $\mathrm{L}=$ $\mathrm{R} ; \mathrm{R}=\mathrm{L}$. gyrus (Figure 3(b)). Individual data showed that these results were found in approximately $70 \%$ of patients.

\section{DISCUSSION}

The aim of the present study was to further investigate the nature of a N400 elicited by incongruity detected in an auditory word-picture pair (Linguistic N400) stimulus and in an environmental sound-picture pair (Non-linguistic N400) stimulus, and to specify the anatomical sources of both N400s. As expected [5,6,22], our data showed a larger N400 in response to incongruent than to congruent stimulus pairs in both tasks, confirming that the N400 is not specific to the detection of lexico-semantic errors but can be elicited by the detection of a broader type of incongruities [26-28,30,84,85]. Shorter peak latencies were found for the Non-linguistic N400, a finding that is consistent with other previous works $[22,23,27,53]$. The observed differences in the N400 latency may stem from a quicker integration of the environmental sound and the corresponding picture due to the less arbitrary character of the relationship with its world-referent [13].

Alternatively, latency differences can be accounted for by the linguistic (or not) nature of the stimuli. Hence, conceptual processing of environmental sounds (ex: sound of yelp) could be faster than linguistic processing (ex: word "dog”) because the access to word meaning is mediated through phonological representation whereas environmental sounds may activate more directly the corresponding semantic representations [22].

Consistent with the literature [5,10,33,54], the scalp topography of the Linguistic N400 showed a centro-parietal distribution with a right-greater-than-left hemispheric asymmetry. In contrast, the Non-linguistic N400 component showed a fronto-central scalp topography with no hemispheric asymmetry. Studies that included environmental sound-picture matching task showed controversial results since some reported a left-greater-than-right cerebral asymmetry [26,30,34] while others found no asymmetry [22,27,28] for the Non-linguistic N400. In the present study, no such hemispheric asymmetry was found.

Latency and topographical differences obtained between Linguistic and Non-linguistic N400s may reflect different processes relying on distinct cerebral generators, but may also be observed when the same cognitive operation is applied to process contexts (for example, images vs. sounds) that differ in their cortical representations [53]. A way to avoid this limitation would have been to use written instead of auditory words in the linguistic task. However, since we wanted to develop a paradigm that is suitable for all populations, including young children and cognitively challenge individuals, we did not want the task to required reading abilities. Another 
way would have been to present the picture prior to environmental sound in the non-linguistic task. However, a pilot study showed that environmental sounds needed to have a significantly longer duration than words to be recognized and that duration greatly varies between sounds, which would have significantly influenced the non-linguistic N400 latency and its variability. Therefore, we cannot exclude that the cognitive process associated with each N400 is the same and that the observed differences are due to the cortical activation preceding/or occurring in parallel and generated by different types of stimuli. Nevertheless, a paired Hotelling T2 test was applied on the data of each task in order to eliminate early components when performing source analyses.

In our study, the picture-auditory word matching task was a French adaptation of the linguistic task used by Connolly et al. [10] that evoked a Linguistic N400 with a tendency for a right-greater-than-left cerebral asymmetry. This topographical pattern has commonly been reported in the literature [5,33], and was also found in the present study for the Linguistic N400. The results of the source analysis for the Linguistic N400 are also similar to those obtained in EEG [44], MEG [40,42,52,59] and fMRI [86] (for a review, see [87]), that all showed left superior temporal gyrus sources for the Linguistic N400. This cerebral region, located in Wernicke's area, is typically associated with phonological and semantic processing and a lesion in this area has been shown to induce comprehensive language deficits such Wernicke's aphasia. The localization of generators in this region is thus consistent with a N400 elicited by the detection of an incongruity occurring during auditory language processing.

Even if no hemispheric asymmetry was found in the Non-linguistic N400 topography, the results of the BMA source analysis suggested a laterality effect, in showing a greater involvement of right than left middle and superior temporal region in processing of the environmental sounds (see Figure 3). These results are compatible with results from brain imaging studies [62,64,66] showing that this region is involved in the processing of environmental sounds. Damage in this area may induce auditory agnosia [88], an auditory impairment affecting the processing of environmental sounds.

Results of the source analyses also revealed generators in the parahippocampal region that were common to both N400 components. The parahippocampal gyrus has been associated with recall activity[89]. The contribution of this region may thus be related to the very nature of the tasks we used which both involved the access to representations that are stored in long-term memory.

In a recent study comparing N400 components elicited by linguistic tasks (semantic and phonologic) and a non-linguistic task (image categorization), Khateb et al. [53] found no topographical or sources analysis differ- ences between tasks. All N400s had a central distribution and originated from the middle and superior temporal gyri. In the present study, we found topographical differences between the Linguistic N400 (centro-parietal distribution) and Non-linguistic N400 (fronto-central distribution). Our source analyses suggested different brain generators for both Linguistic and Non-linguistic N400s, located respectively in the left superior temporal gyrus and in the right middle and superior temporal gyri. Differences between the present findings and results from Khateb et al. [53] may be explained by methodological differences. First, different tasks were used to elicit the Non-linguistic N400 components. It can be hypothesized that the environmental sound-picture matching task used in our experiment induces less verbalization than the image categorization task used in Khateb et al.' study [53]. This could explain the fronto-central distribution associated with the Non-linguistic N400 and its right middle and superior temporal gyri origin found in our study. The fronto-central distribution could also be explained by a familiarity effect and/or the implication of the attention network. It is noteworthy that such a fronto-central distribution has also been reported in previous studies using environmental sound tasks [22,23,27,30]. Second, differences in source analysis approaches may also partly explain differences between our results and those of Khateb et al. [53].

In the present study, we used a unique and powerful source analyses model, the BMA approach, including LORETA method. This is the first study investigating Linguistic N400 cerebral generators using the BMA approach. Most distributed inverse solutions (DIS) give quite good results when dealing with widespread activities. However, they fail to recover spatially concentrated sources due to their tendency to smooth out activations. The most common procedure followed by any DIS is, for any given mathematical constraint, to assume that the possible generators are confined to a given region of the brain and to make all statistical inferences conditional on that assumption. However, different choices of the anatomical constraints lead to completely different current density distributions. This introduces some uncertainty about the model assumptions, which must be taken into account. This problem, commonly omitted by traditional inverse solution methods, has been widely treated in the Bayesian Inference literature and it is known as Model Uncertainty. The BMA approach used in this study [60] is based on the application of the Bayesian model inference scheme [67] under the evidence approximation [68], to the EEG Inverse Problem. This provides a coherent mechanism for accounting for the type of model uncertainty described above. The BMA has shown significantly less blurring and ghost sources than alternative distributed inverse solution approaches and is significantly less affected by the underestimation of deep 
sources.

Data set collected in both tasks and conditions comprised a fairly high proportion of artifact-free trials. This was obtained by presenting each stimulus set twice. It is worth mentioning that we did not find the repetition effect reported by some authors [82,83,90,91] when measuring N400 elicited by the detection of incongruity in sentence stimuli. These authors showed that the N400 amplitude was reduced in the second presentation of the sentence stimulus set. According to these authors, the last word of a sentence gets more predictable at the second or multiple presentations. This predictability elicits a diminution of the N400 amplitude. In the present study, we used pictures, auditory words and environmental sounds. These stimuli may not provide a semantic context that is sufficiently rich to contribute in reducing the level of incongruity detected by the subjects at the second presentation.

Within the present set of experiments, it may be argued that the Non-linguistic N400 was elicited because the subjects verbalized the environmental sounds before the appearance of the picture, thereby eliciting a Linguistic N400. To address this issue, Aramaki et al. [92] only used sounds in their experimental design. However, this was not possible in the present study. In order to minimize this effect, all participants performed the environmental sound-picture matching task prior to the picture-auditory word matching task. In a pilot study with no EEG recording, we investigated the order effect on the performance of a small group of subjects and found that all participants obtained very high scores in both tasks (ceiling effect), with no task order effect. In addition, responses given by the participants questioned after the testing do not support the hypothesis of the verbalization during the environmental sound-picture matching task. Indeed, participants reported they were adopting different strategies when performing the picture-auditory word or the environmental sound-picture matching task, i.e., silently anticipating the word congruent with the picture prime in the picture-auditory word matching task, and visualizing the object or an environmental scene eliciting such a sound in the environmental sound-picture matching task. Thus, it can be hypothesized that the picture-auditory word matching task involved a verbalization of the name of the picture presented before the word onset, while the environmental sound-picture matching task induced a visual representation of the environmental sound presented before the picture onset. The fact that an activation of the visual associative cortex (left lingual gyrus) was found only in the environmental sound-picture task brings additional support to this interpretation.

\section{CONCLUSION}

The present paper is the first study that proposes models of cerebral generators for both the Non-linguistic N400, generated by an environmental sound-picture matching task, and the Linguistic N400, elicited by a pictureauditory word matching task, in healthy adults. Results for source analysis indicated that BMA approach is an adequate model for the localization of the N400. Findings of the present study concur with brain imaging data and single case studies suggesting that linguistic incongruities involve Wernicke's area, whereas those related to environmental sounds recruit the right temporal region. Our results are also consistent with those of a PET study conducted by Thierry and Price [65], who reported activation in the left superior temporal sulcus and left ventral inferior frontal gyrus in the processing of the linguistic incongruity, whereas the right midfusiform and right posterior middle temporal gyrus were activated during the processing of non-linguistic incongruity. Interestingly, hemispheric dissociation in processing linguistic and nonlinguistic material was obtained regardless of the modality of presentation (visual or auditory). It thus appears that the stimulus type, linguistic vs. non-linguistic, influences which cerebral regions will be recruited in detecting semantic incongruity. This suggests the existence of two parallel pathways for the processing of linguistic and non-linguistic incongruities.

\section{ACKNOWLEDGEMENTS}

We wish to thank Yanays Rodriguez, Alejandro Ojeda, and Eduardo Aubert for helping with source analyses and providing fruitful discussions. We are also indebted to Martin Thériault, Benoît Gagnon and Julie Senécal for programming tasks and helping in generating stimuli. This work was supported by by the Canada Research Chair in Developmental Neuropsychology held by Dr. Lassonde, and scholarships from the Canadian Institutes of Health Research (CIHR) and the Fonds de la Recherche du Québec - Santé (FRQS) awarded to Dr. Gallagher.

\section{REFERENCES}

[1] Brandeis, D. and Lehmann, D. (1986) Event-related potentials of the brain and cognitive processes: Approaches and applications. Neuropsychologia, 24, 151-168. http://dx.doi.org/10.1016/0028-3932(86)90049-7

[2] Auchterlonie, S., Phillips, N.A., Chertkow, H., 2002. Behavioural and electrical brain measures of semantic priming in patients with Alzheimer's disease: Implications for access failure versus deterioration hypotheses. Brain and Cognition, 48, 264-267.

[3] Kutas, M., Federmeier, K.D. (2011) Thirty years and counting: Finding meaning in the N400 component of the event-related brain potential (ERP). Annual Review of Psychology, 62, 621-647.

http://dx.doi.org/10.1146/annurev.psych.093008.131123

[4] Phillips, N.A. and Lesperance, D. (2003) Breaking the waves: Age differences in electrical brain activity when 
reading text with distractors. Psychology and Aging, 18, 126-139. http://dx.doi.org/10.1037/0882-7974.18.1.126

[5] Kutas, M. and Hillyard, S.A. (1980) Event-related brain potentials to semantically inappropriate and surprisingly large words. Biological Psychology, 11, 99-116. http://dx.doi.org/10.1016/0301-0511(80)90046-0

[6] Kutas, M. and Hillyard, S.A. (1980) Reading senseless sentences: Brain potentials reflect semantic incongruity. Science, 207, 203-205. http://dx.doi.org/10.1016/0301-0511(80)90046-0

[7] Kutas, M. and Hillyard, S.A. (1984) Brain potentials during reading reflect word expectancy and semantic association. Nature, 307, 161-163. http://dx.doi.org/10.1038/307161a0

[8] Holcomb, P.J. (1993) Semantic priming and stimulus degradation: Implications for the role of the N400 in language processing. Psychophysiology, 30, 47-61. http://dx.doi.org/10.1111/j.1469-8986.1993.tb03204.x

[9] Kutas, M. and Hillyard, S.A. (1989) An electrophysiological probe of incidental semantic association. Journal of Cognitive Neuroscience, 1, 38-49. http://dx.doi.org/10.1162/jocn.1989.1.1.38

[10] Connolly, J.F., Byrne, J.M. and Dyman, C.A. (1995) Assessing adult receptive vocabulary with event-related potentials: An investigation of cross-modal and cross-form priming. Journal of Clinical and Experimental Neuropsychology, 17, 548-565. http://dx.doi.org/10.1080/01688639508405145

[11] Bernadis, P., Salillas, E. and Caramelli, N. (2008) Behavioural and neurophysiological evidence of semantic interaction between iconic gestures and words. Cogn $\mathrm{Neu}$ ropsychology, 25, 1114-1128.

http://dx.doi.org/10.1080/02643290801921707

[12] Dunn, L.M. and Dunn, L.M. (1981) Peabody picture vocabulary test-revised. American Guidance Service, Circle Pines.

[13] Ganis, G., Kutas, M. and Sereno, M.I. (1996) The search for "common sense": An electrophysiological study of the comprehension of words and pictures in reading. Journal of Cognitive Neuroscience, 8, 89-106. http://dx.doi.org/10.1162/jocn.1996.8.2.89

[14] Holcomb, P.J. and McPherson, W.B. (1994) Event-related potentials reflect semantic priming in an object decision task. Brain and Cognition, 24, 259-276. http://dx.doi.org/10.1006/brcg.1994.1014

[15] McPherson, W.B. and Holcomb, P.J. (1999) An electrophysiological investigation of semantic priming with pictures of real objects. Psychophysiology, 36, 53-65 http://dx.doi.org/10.1017/S0048577299971196

[16] Barrett, S.E. and Rugg, M.D. (1989) Event-related potentials and the semantic matching of faces. Neuropsychologia, 27, 913-922. http://dx.doi.org/10.1016/0028-3932(89)90067-5

[17] Bobes, M.A., Valdes-Sosa, M. and Olivares, E (1994) An ERP study of expectancy violation in face perception. Brain and Cognition, 26, 1-22. http://dx.doi.org/10.1006/brcg.1994.1039

[18] Jemel, B., et al. (1999) Event-related potentials to struc- tural familiar face incongruity processing. Psychophsiology, 36, 437-452.

http://dx.doi.org/10.1006/brcg.1994.1039

[19] Olivares, E. I., Saavedra, C., Trujillo-Barreto, N. J. and Iglesias, J. (2013) Long-term information and distributed neural activation are relevant for the "internal features advantage” in face processing: Electrophysiological and source reconstruction evidence. Cortex, 49, 2735-2747. http://dx.doi.org/10.1016/j.cortex.2013.08.001

[20] Jost, K., Hennighausen, E. and Rosler, F. (2004) Comparing arithmetic and semantic fact retrieval: Effects of problem size and sentence constraint on event-related brain potentials. Psychophysiology, 41, 46-59. http://dx.doi.org/10.1111/1469-8986.00119_41_1

[21] Szucs, D. and Soltész, F. (2010) Event-related brain potentials to violations of arithmetic syntax represented by place value structure. Biological Psychology, 84, 354-367. http://dx.doi.org/10.1016/j.biopsycho.2010.04.002

[22] Cummings, A., Čeponienè, R., Koyama, A., Saygin, A.P., Townsend, J. and Dick, F. (2006) Auditory semantic networks for words and natural sounds. Brain Research, 1115, 92-107. http://dx.doi.org/10.1016/j.brainres.2006.07.050

[23] Cummings, A., Čeponienè, R., Dick, F., Saygin, A.P. and Townsend, J. (2008) A developmental ERP study of verbal and non-verbal semantic processing. Brain Research, 1208, 137-149.

http://dx.doi.org/10.1016/j.brainres.2008.02.015

[24] Fogelson, N., Loukas, C., Brown, J. and Brown, P. (2004) A common N400 EEG component reflecting contextual integration irrespective of symbolic form. Clinical Neurophysiology, 115, 1349-1358. http://dx.doi.org/10.1016/j.clinph.2004.01.010

[25] Kirmse, U., Jacobsen, T. and Schröger, E. (2009) Familiarity affects environmental sound processing outside the focus of attention: an event-related potential study. Clinical Neurophysiology, 120, 887-896. http://dx.doi.org/10.1016/j.clinph.2009.02.159

[26] Lebrun, N., et al. (2001) An ERD mapping study of the neurocognitive processes involved in the perceptual and semantic analysis of environmental sounds and words. Cognitive Brain Research, 11, 235-248. http://dx.doi.org/10.1016/S0926-6410(00)00078-1

[27] Orgs, G., Lange, K., Dombrowski, J.H. and Heil, M. (2006) Conceptual priming for environmental sounds and words: an ERP study. Brain and Cognition, 62, 267-272. http://dx.doi.org/10.1016/j.bandc.2006.05.003

[28] Orgs, G., Lange, K., Dombrowski, J.H. and Heil, M. (2008) N400-effects to task-irrelevant environmental sounds: Further evidence for obligatory conceptual processing. Neuroscience Letters, 436, 133-137. http://dx.doi.org/10.1016/j.neulet.2008.03.005

[29] Polich, J. (1985) N400s from sentences, semantic categories, number and letter strings. Bulletin of the Psychonomic Society, 23, 361-364. http://dx.doi.org/10.3758/BF03330184

[30] Van Petten, C. and Rheinfelder, H. (1995) Conceptual relationships between spoken words and environmental 
sounds: Event-related brain potential measures. Neuropsychologia, 33, 485-508.

http://dx.doi.org/10.1016/0028-3932(94)00133-A

[31] Grigor, J., Van, S., Toller, Behan, J. and Richardson, A. (1999) The effect of odour priming on long latency visual evoked potentials of matching and mismatching objects. Chemical Senses, 24, 137-144. http://dx.doi.org/10.1093/chemse/24.2.137

[32] Sarfarazi, M., Cave, B., Richardson, A., Behan, J. and Sedgwick, E.M. (1999) Visual event related potentials modulated by contextually relevant and irrelevant olfactory primes. Chemical Senses, 24, 145-154. http://dx.doi.org/10.1093/chemse/24.2.145

[33] Kutas, M. and Hillyard, S.A. (1982) The lateral distribution of event-related potentials during sentence processing. Neuropsychologia, 20, 579-590. http://dx.doi.org/10.1016/0028-3932(82)90031-8

[34] Plante, E., Van Petten, C. and Senkfor, A.J. (2000) Electrophysiological dissociation between verbal and nonverbal semantic processing in learning disabled adults. Neuropsychologia, 38, 1669-1684. http://dx.doi.org/10.1016/S0028-3932(00)00083-X

[35] De Munck, J.C., van Dijk, B.W. and Spekeijse, H. (1988) Mathematical dipoles are adequate to describe realistic generators of human brain activity. IEEE Transactions on Biomedical Engineering, 35, 960-966. http://dx.doi.org/10.1109/10.8677

[36] Nunez, P.L. (1981) A study of origins of the time dependencies of scalp EEG: I-theoretical basis. IEEE Transactions on Biomedical Engineering, 28, 271-280. http://dx.doi.org/10.1109/TBME.1981.324700

[37] Scherg, M. and von Cramon, D. (1986) Evoked dipole source potentials of the human auditory cortex. Electroencephalography and Clinical Neurophysiology, 65, 344360. http://dx.doi.org/10.1016/0168-5597(86)90014-6

[38] Scholz, B. and Schwierz, G. (1994) Probability-based current dipole localization from biomagnetic fields. IEEE Transactions on Biomedical Engineering, 41, 735-742. http://dx.doi.org/10.1109/10.310089

[39] Luck, S.J. (2005) An introduction to the event-related potential technique. MIT Press, Cambridge.

[40] Helenius, P., Salmelin, R., Service, E. and Connolly, J.F. (1998) Distinct time courses of word and context comprehension in the left temporal cortex. Brain, 121, 11331142. http://dx.doi.org/10.1093/brain/121.6.1133

[41] Helenius, P., Salmelin, R., Service, E. and Connolly, J.F. (1999) Semantic cortical activation in dyslexic readers. Journal of Cognitive Neuroscience, 11, 535-550. http://dx.doi.org/10.1162/089892999563599

[42] Simos, P.G., Basile, L.F.H. and Papanicolaou, A.C. (1997) Source localization of the N400 response in a sentencereading paradigm using evoked magnetic fields and magnetic resonance imaging. Brain Research, 762, 29-39. http://dx.doi.org/10.1016/S0006-8993(97)00349-1

[43] Mäkela, A.M., Mäkinen, V., Nikkilä, M., Ilmoniemi, R.J. and Tiitinen, H. (2001) Magnetoencephalographic (MEG) localization of the auditory N400m: Effects of stimulus duration. Neuroreport, 12, 249-253. http://dx.doi.org/10.1097/00001756-200102120-00014

[44] D’Arcy, R.C.N., Connolly, J.F., Service, E., Hawco, C.S. and Houlihan, M.E. (2004) Separating phonological and semantic processing in auditory sentence processing: A high-resolution event-related brain potential study. $\mathrm{Hu}$ man Brain Mapping, 22, 40-51.

http://dx.doi.org/10.1002/hbm.20008

[45] D’Arcy, R.C.N., Service, E., Connolly, J.F. and Hawco, C.S. (2005) The influence of increased working memory load on semantic neural systems: A high-resolution eventrelated potential study. Cognitive Brain Research, 22, 177-191. http://dx.doi.org/10.1016/j.cogbrainres.2004.08.007

[46] Dien, J., Michelson, C.A. and Franklin, M.S. (2010) Separating the visual sentence N400 effect from the P400 sequential expectancy effect: Cognitive and neuroanatomical implications. Brain Research, 1355, 126-140. http://dx.doi.org/10.1016/j.brainres.2010.07.099

[47] Hamm, J.P., Johnson, B.W. and Kirk, I.J. (2002) Comparison of the N300 and N400 ERPs to picture stimuli in congruent and incongruent contexts. Clinical Neurophysiology, 113, 1339-1350.

http://dx.doi.org/10.1016/S1388-2457(02)00161-X

[48] Silva-Pereyra, J., Rivera-Gaxiola, M., Aubert, E., Bosch, J., Galan, L. and Salazar, A. (2003) N400 during lexical decision tasks: A current source localization study. Clinical Neurophysiology, 114, 2469-2486. http://dx.doi.org/10.1016/S1388-2457(03)00248-7

[49] Bölte, J., Schulz, C. and Dobel, C. (2010) Processing of existing, synonymous, and anomalous German derived adjectives: An MEG study. Neuroscience Letters, 469, 107-111. http://dx.doi.org/10.1016/j.neulet.2009.11.054

[50] Giglio, A.C., Minati, L. and Boggio, P.S. (2013) Throwing the banana away and keeping the peel: Neuroelectric responses to unexpected but physically feasible action endings. Brain Research, 1532, 56-62. http://dx.doi.org/10.1016/j.brainres.2013.08.017

[51] Haan, H., Streb, J., Bien, S. and Rosler, F. (2000) Individual cortical current density reconstructions of the semantic N400 effect: Using a generalized minimum norm model with different constraints (L1 and L2 norm). $\mathrm{Hu}$ man Brain Mapping, 11, 178-192.

http://dx.doi.org/10.1002/1097-0193(200011)11:3<178:: AID-HBM40>3.0.CO;2-0

[52] Halgren, E., Dhond, R.P., Christensen, N., Van Petten, C., Marinkovic, K., Lewine, J.D. and Dale, A.M. (2002) N400-like magnetoencephalography response modulated by semantic context, word frequency, and lexical class in sentences. Neuroimage, 17, 1101-1116. http://dx.doi.org/10.1006/nimg.2002.1268

[53] Khateb, A., Pegna, A.J., Landis, T., Mouthon, M.S. and Annoni, J.M. (2010) On the origin of the N400 effects: An ERP waveform and source localization analysis in three matching tasks. Brain Topography, 23, 311-320. http://dx.doi.org/10.1007/s10548-010-0149-7

[54] Khateb, A., Pegnaa, A.J., Landisc, T., Micheld, C.M., Brunetd, D., Seghierf, M.L. and Annoni, J.M. (2007) Rhyme processing in the brain: An ERP mapping study. International Journal of Psychophysiology, 63, 240-250. 
http://dx.doi.org/10.1016/j.ijpsycho.2006.11.001

[55] Proverbio, A.M., Crotti, N., Zani, A. and Adomi, R. (2009) The role of left and right hemispheres in the comprehension of idiomatic language: An electrical neuroimaging study. BMC Neuroscience, 10, 116. http://dx.doi.org/10.1186/1471-2202-10-116

[56] Qiu, J., Li, H., Chen, A. and Zhang, Q. (2008) The neural basis of analogical reasoning: An event-realted potential study. Neuropsychologia, 46, 3006-3013. http://dx.doi.org/10.1016/j.neuropsychologia.2008.06.008

[57] Ren, G.Q., Liu, Y. and Han, Y.C. (2009) Phonological activation in chinese reading: An event-related potential study using low-resolution electromagnetic tomography. Neuroscience, 164, 1623-1631. http://dx.doi.org/10.1016/j.neuroscience.2009.09.029

[58] Schulz, E., Maurer, U., van der Mark, S., Bucher, K., Brem, S., Martin, E. and Brandeis D. (2008) Impaired semantic processing during sentence reading in children with dyslexia: Combined fMRI and ERP evidence. Neuroimage, 41, 153-168.

http://dx.doi.org/10.1016/j.neuroimage.2008.02.012

[59] Travis, K.E., et al. (2013) Independence of early speech processing from word meaning. Cerebral Cortex, 23, 2370-2379. http://dx.doi.org/10.1093/cercor/bhs228

[60] Trujillo-Barreto, N.J., Aubert-Vázquez, E. and Valdés-Sosa, P.A. (2004) Bayesian Model Averaging in EEG/MEG imaging. Neuroimage, 21, 1300-1319. http://dx.doi.org/10.1016/j.neuroimage.2003.11.008

[61] Kotz, S.A., Opitz, B. and Friederici, A.D. (2007) ERP effects of meaningful and non-meaningful sound processing in anterior temporal patients. Restorative Neurology and Neuroscience, 25, 273-284.

[62] Thierry, G., Giraud, A.L. and Price, C. (2003) Hemispheric dissociation in access to the human semantic system. Neuron, 38, 499-506. http://dx.doi.org/10.1016/S0896-6273(03)00199-5

[63] Schnider, A., Benson, D.F., Alexander, D.N. and SchniderKlaus, A. (1994) Non-verbal environmental sound recognition after unilateral hemispheric stroke. Brain, 17, 281287. http://dx.doi.org/10.1093/brain/117.2.281

[64] Opitz, B., Mecklinger, A. and Friederici, A.D. (2006) Functional asymmetry of human prefrontal cortex: Encoding and retrieval of verbally and nonverbally coded information. Learning Memory, 7, 85-96. http://dx.doi.org/10.1101/lm.7.2.85

[65] Thierry, G. and Price, C. (2006) Dissociating verbal and nonverbal conceptual processing in the human brain. Journal of Cognitive Neuroscience, 18, 1018-1028. http://dx.doi.org/10.1162/jocn.2006.18.6.1018

[66] Zatorre, R.J., Bouffard, M. and Belin, P. (2004) Sensitivity to auditory object features in human temporal neocortex. Journal of Neuroscience, 24, 3637-3642. http://dx.doi.org/10.1523/JNEUROSCI.5458-03.2004

[67] Hoeting, J.A., Madigan, D., Raftery, A.E. and Volinsky, C.T. (1999) Bayesian model averaging: A tutorial. Statistical Science, 14, 382-417.

[68] MacKay, D.J.C. (1992) Bayesian interpolation. Neural Computation, 4, 415-447. http://dx.doi.org/10.1162/neco.1992.4.3.415

[69] Pascual-Marqui, R.D., Michel, C.M. and Lehmann, D. (1994) Low resolution electromagnetic tomography: A new method for localizing electrical activity in the brain. International Journal of Psychophysiology, 18, 49-65. http://dx.doi.org/10.1016/0167-8760(84)90014-X

[70] Dunn, L.M., Thériault-Whalen, C.M. and Dunn, L.M. (1993) Échelle de vocabulaire en images Peabody. PSYCAN, Toronto.

[71] Alario, F.X. and Ferrand, L. (1999) A set of 400 pictures standardized for French: Norms for name agreement, image agreement, familiarity, visual complexity, image variability, and age of acquisition. Behavior Research Methods, 31, 531-552.

[72] Bonin, P., Peereman, R., Malardier, N., Méot, A. and Chalard, M. (2003) A new set of 299 pictures standardized for French for name agreement, image agreement, conceptual familiarity, visual complexity, image variability and age of acquisition. Behavior Research Methods, 35, 158-167.

[73] Chalard, M., Bonin, P., Méot, A., Boyer, B. and Fayol, M. (2003) Objective age-of-acquisition (AoA) norms for a set of 230 object names in French: Relationship with psycholinguistic variables, the English data from Morrison et al. (1997), and naming latencies. European Journal of Cognitive Psychology, 15, 209-245.

http://dx.doi.org/10.1080/09541440244000076

[74] Ferrand, L., Grainger, J. and New, B. (2002) Normes d'âge d'acquisition pour 400 mots monosyllabiques. Notes méthodologiques sur www.lexique.org

[75] Gratton, G., Coles, M.G., Donchin, E. (1983) A new method for off-line removal of ocular artifact. Electroencephalography and Clinical Neurophysiology, 55, 468-484. http://dx.doi.org/10.1016/0013-4694(83)90135-9

[76] McCarthy, G. and Wood, C.C. (1985) Scalp distribution of event-related potentials: An ambiguity associated with analysis of variance models. Electroencephalography and Clinical Neurophysiology, 62, 203-208. http://dx.doi.org/10.1016/0168-5597(85)90015-2

[77] Urbach, T.P. and Kutas, M. (2006) Interpreting eventrelated brain potential (ERP) distributions: Implications of baseline potentials and variability with application to amplitude normalization by vector scaling. Biological Psychology, 72, 333-343.

http://dx.doi.org/10.1016/j.biopsycho.2005.11.012

[78] Rush, S. and Driscoll, D.A. (1969) EEG electrode sensitivity-An application of reciprocity. IEEE Transactions on Biomedical Engineering, 16, 15-22. http://dx.doi.org/10.1109/TBME.1969.4502598

[79] Riera, J.J., Fuentes, M.E., Valdes, P.A. and Oharriz, Y. (1997) Theoretical basis of the EEG spline inverse solutions for spherical head model. Biomedical Technology, 42, 219-222.

[80] Riera, J., Valdes, P., Fuentes, M.E. and Oharriz, Y. (1997) Explicit Backus and Gilbert EEG inverse solution for spherical head model. Biomedical Technology, 42, 216218.

[81] Benjamini, Y. and Hochberg, Y. (1995) Controlling the 
false discovery rate: A practica1 and powerful approach to multiple testing. Royal Statistic Society Series B (Methodological), 57, 289-300.

[82] Besson, M. and Kutas, M. (1993) The many facets of repetition: A cued-recall and event-related potential analysis of repeating words in same versus different sentence contexts. Journal of Experimental Psychology: Learning Memory and Cognition, 19, 1115-1133. http://dx.doi.org/10.1037/0278-7393.19.5.1115

[83] Besson, M., Kutas, M. and Van Petten, C. (1992) An event-related potential (ERP) analysis of semantic congruity and repetition effects in sentences. Journal of Cognitive Neuroscience, 4, 132-149.

http://dx.doi.org/10.1162/jocn.1992.4.2.132

[84] Daltrozzo, J. and Schön, D. (2009) Conceptual processing in music as revealed by N400 effects on words and musical targets. Journal of Cognitive Neuroscience, 21, 18821892. http://dx.doi.org/10.1162/jocn.2009.21113

[85] Painter, J.G. and Koelsch, S. (2011) Can out-of-context musical sounds convey meaning? An ERP study on the processing of meaning in music. Psychophysiology, 48, 645-655.

http://dx.doi.org/10.1111/j.1469-8986.2010.01134.x

[86] Willems, R.M., Ozyurek, A. and Hagoort, P. (2008) Seeing and Hearing Meaning: ERP and fMRI Evidence of Word versus Picture Integration into a Sentence Context. Journal of Cognitive Neuroscience, 20, 1235-1249.

http://dx.doi.org/10.1162/jocn.2008.20085
[87] Lau, E.F., Phillips, C. and Poeppel, D. (2008) A cortical network for semantics: (de)constructing the N400. Nature Reviews. Neuroscience, 9, 920-933.

[88] Fujii, T., Fukatsu, R., Watabe, S., Ohnuma, A., Teramura, K., Kimura, I., Saso, S. and Kogure, K. (1990) Auditory sound agnosia without aphasia following a right temporal lesion. Cortex, 26, 263-268. http://dx.doi.org/10.1016/S0010-9452(13)80355-3

[89] Alkire, M.T., Haier, R.J., Fallon, J.H. and Cahill, L. (1998) Hippocampal, but not amygdala, activity at encoding correlates with long-term, free recall of nonemotional information. PNAS, 95, 14506-14510.

[90] Young, M.P. and Rugg, M.D. (1992) Word frequency and multiple repetition as determinants of the modulation of event-related potentials in a semantic classification task. Psychophysiology, 29, 664-676. http://dx.doi.org/10.1111/j.1469-8986.1992.tb02044.x

[91] Doyle, M.C., Rugg, M.D. and Wells, T. (1996) A comparison of the electrophysiological effects of formal and repetition priming. Psychophysiology, 33,132-147. http://dx.doi.org/10.1111/j.1469-8986.1996.tb02117.x

[92] Aramaki, M., Marie, C., Kronland-Martinet, R., Ystad, S. and Besson, M. (2010) Sound categorization and conceptual; priming for nonlinguistic ans linguistic sounds. Journal of Cognitive Neuroscience, 22, 2555-2569. http://dx.doi.org/10.1162/jocn.2009.21398 